\title{
Sélection de variétés performantes de riz pluvial (Oryza sp.) dans la région subéquatoriale du Congo Brazzaville
}

\author{
A. S. P. Nguetta ${ }^{1 *}$, J. Y. Lidah', C. N. M. Ebélébé ${ }^{3}$ et R. G. Guéi ${ }^{2}$ \\ 'UFR Biosciences, Laboratoire de génétique, Université de Cocody - Abidjan, \\ 22 B.P. 582 Abidjan 22, Côte d'Ivoire \\ ${ }^{2}$ Association pour le développement de la riziculture en Afrique de l'Ouest (ADRAO), \\ Ol B. P. 2551 Bouaké 01, Côte d'lvoire \\ ${ }^{3}$ Université Marien Ngouabi de Brazzaville, Institut de développement rural(IDR), \\ Département de cycle moyen et professionnel. B.P. 69 Brazzaville, République du Congo
}

(Reçu le 03 Février 2006, accepté le 10 Juin 2006)

*Correspondance,courriel : nguettaewatty@yahoo.fr

\section{Résumé}

Pour résoudre le problème de déficit et de dépendance vis-à-vis de l'extérieur, les expérimentations ont été menées dans la région Nord du Congo Brazzaville pour sélectionner des variétés performantes de riz pluvial.

Dix huit variétés de riz issues de la collection de l'Association pour le Développement de la Riziculture en Afrique de l'Ouest (ADRAO), Côte d'Ivoire ont été évaluées. Chaque variété a été semée en utilisant un dispositif en blocs complètement randomisés avec trois répétitions en 2003 et 2004 . Les caractères agronomiques tels que la densité à la levée, la densité au tallage, le nombre total de talles, le nombre de talles fertiles, la période de $50 \%$ floraison, la taille des plantes à maturité, la verse, la résistance aux maladies ainsi que le rendement ont été mesurés.

Toutes les variétés testées se sont adaptées dans la région à des degrés divers. Cependant, trois variétés ont pu être sélectionnées parmi les autres au cours de cette expérimentation. II s'agit de WAB 450-24-I-B-P33-HB, WAB 32-55 et WAB 377-B-5-HI qui ont des rendements nettement plus élevés que les autres. Ces rendements ont été respectivement de 7.03, 5.10 et 4.30 tonnes à l'hectare. Les résultats de criblage ont en plus montré que ces variétés ont été moins affectées par la pyriculariose et n'ont pas subi de verse.

Mots-clés : riz pluvial, talle, variété, rendement, ADRAO, Congo Brazzaville. 


\section{Abstract \\ Selection of rice (Oryza sp.) elites upland varieties in the subequatorial region of Congo Brazzaville}

To assess the problem of dependency on other countries for rice production, fields' studies were conducted in the north part of the republic of Congo Brazzaville to identify high yield upland varieties.

The 18 varieties evaluated in this experiment were obtained from West Africa Rice Development Association (WARDA) collection, Côte d'Ivoire. Each variety was grown in replicated trials at SADC production centre in 2003 and 2004 in the District of OYO. The agronomic characters such as the number of total tillers, the number of total fertile tillers, the plants height at maturity, the period of $50 \%$ flowering, lodging, disease resistance and yield were measured.

Each one of the tested varieties was adapted to the region at different levels. However, three varieties may be selected among the others. These varieties are WAB 450-24-I-B$P 33-H B, W A B$ 377-B-5-HI and $W A B$ 32-55 which presented high yield compared to the others. The yields were respectively, $7.03,4.30$ and $5.10 \mathrm{t} / \mathrm{ha}$. The results also showed that these varieties were less affected by diseases and did not lodge.

Keywords : rice Upland., tiller, variety, yield, WARDA, Congo Brazzaville.

\section{Introduction}

Le riz (Oryza sp.) constitue la source d'alimentation pour environ $50 \%$ de la population mondiale [1,2]. Avec une croissance estimée à 600000 ha / an, les cultures du riz ont atteint 150 millions d'hectares et une production de 395 millions de tonnes de paddy en 2003 ; cependant $90 \%$ de la production mondiale en riz sont fournis par l'Asie Orientale et Méridionale avec $33 \%$ pour la Chine [3-5].

Les principaux pays exportateurs mondiaux sont la Thaïlande, le Vietnam, la Chine, l'Inde et les Etats-Unis tandis que les pays importateurs se situent surtout en Afrique subsaharienne et au Moyen-Orient $[5,6]$.

Dans divers pays d'Afrique tropicale, le riz tend à se substituer à d'autres céréales tels que le mil et le sorgho en particulier [7]. La demande du riz en Afrique de l'0uest et du Centre crôit de $6 \%$ par an. Cette demande est liée principalement à la croissance démographique et à l'accroissement de la part du riz dans le régime alimentaire [8]. Cependant, $60 \%$ des besoins en riz de l'Afrique sont couverts par les importations parce que la production locale reste largement insuffisante [8]. 
Au niveau de la République du Congo Brazzaville, le riz représente en effet, la denrée alimentaire la plus importante après le manioc. Les besoins nationaux annuels sont estimés à 31000 tonnes. En 1998, plus de 20000 tonnes de riz ont été importées avec les réserves financières nationales estimées à 10 milliards de francs CFA [9].

Malgré la demande de plus en plus croissante du riz, la République du Congo Brazzaville est pratiquement le seul pays en Afrique Centrale qui dépend exclusivement de l'extérieur pour satisfaire ses besoins alimentaires [10]. Le gouvernement congolais n'a pas encore mis en place une bonne politique agricole conduisant à la production de cultures vivrières tel que le riz [5].

Pour corriger cette situation de déficit alimentaire, réduire l'importation du riz et remédier à l'insécurité alimentaire croissante, la production du riz en grande quantité s'avère une priorité nationale [11]. Ainsi, le gouvernement de la République du Congo a entrepris à travers l'ADRAO et la société pétrolière ENI Congo, le développement d'un " projet pilote ॥ de la filière riz dans la partie Nord du pays. Ce projet pilote qui est un modèle d'exploitation rizicole dans le pays sera reproduit dans d'autres districts et régions pour l'augmentation de la production de riz au Congo Brazzaville.

L'objectif principal de cette étude est la sélection des variétés très performantes de riz, produites par l'ADRA0, qui s'adaptent dans la région Nord du Congo Brazzaville.

\section{Matériel et méthodes}

\section{2-1. Site d'expérimentation}

Cette étude a été menée en 2003 et 2004 dans le district de 0yo, dans la région de la cuvette de la République du Congo, à environ $450 \mathrm{~km}$ au Nord de Brazzaville.

La mise en place a été réalisée sur la station de production de la Société Agricole et de Développement de la Cuvette (SADC) située à $M B O B O$, à $40 \mathrm{~km}$ au sud du district de 0 yo sur un sol sablo-limoneux avec très peu d'argile. Ce sol présente une très grande porosité et est pauvre en matière organique mais très profond avec une assez bonne activité biologique. Ce sont des sols noirs tropicaux et alluvionnaux d'une savane inondée saisonnière [12].

Le climat est de type subéquatorial avec deux saisons pluvieuses et deux saisons sèches. Les expériences ont été mises en place pendant la grande saison des pluies de septembre à décembre 2003 et au cours de la petite saison pluvieuse de mars à mai 2004. La pluviométrie moyenne observée au cours de 2003 et 2004 est de $1800 \mathrm{~mm}$ avec plus de 115 jours de pluie et une température moyenne de $26{ }^{\circ} \mathrm{C}$. 


\section{2-2. Le matériel végétal}

Le matériel végétal est constitué de 18 variétés élites de riz pluvial dont WAB 99-12, WAB 450-24-I-B-P33-HB, WAB 32-55, WAB 450-I-B-P24-HB, WAB 224-12-HB, WAB 450-I-BPI60-HB (NERICA 6), WAB 450-I-B-P91-HB (NERICA 4), WAB 450-I-B-P20-HB (NERICA 7), WAB 515-B-35A-1-1, WAB 306-B-B-L L-L L-HB, WAB 450-11-1-1-P31-1-HB (NERICA 2), WAB 450-111-1-P50-HB, WAB 337-B-B-20-H4, WAB 570-10-1A-1-10, WAB 377-B-5-HI, WAB 99-H-16-HB, WAB 99-2-1 et WAB 365-B-6- $\mathrm{H}_{2}-\mathrm{HB}$, provenant de la collection de l'ADRAO. Ce sont des variétés hybrides très performantes dont la plupart sont issues du croisement interspécifique entre le riz asiatique Oryza sativa L. et le riz africain Oryza glaberrima Steud. L'espèce Oryza sativa a une production très élevée mais est susceptible à beaucoup de maladies alors que l'espèce 0 . glaberrima a une production faible mais est résistante à la plupart des maladies du riz.

\section{2-3. Pratique culturale}

Le semis des 18 variétés a été réalisé manuellement sur une superficie totale de $1053 \mathrm{~m}^{2}$. Le dispositif en blocs randomisés avec trois répétitions a été utilisé pour la mise en place de toutes les parcelles.

Les dimensions d'un bloc sont de $9 \mathrm{~m}$ de large sur $39 \mathrm{~m}$ de long, soit une superficie de $351 \mathrm{~m}^{2}$. Dans chaque bloc, les parcelles élémentaires sont de $4 \mathrm{~m}$ de long et de $3 \mathrm{~m}$ de large, soit une superficie de $12 \mathrm{~m}^{2}$. Le semis est en ligne avec un écartement interligne de $20 \mathrm{~cm}$ et intraligne de $20 \mathrm{~cm}$ entre poquets.

Le sol a été fertilisé au moment du semis avec un engrais de fond, le N.P.K.: 17-17-17 à la dose de $250 \mathrm{~kg} / \mathrm{ha}$. Deux apports supplémentaires d'azote sous forme d'urée ont été effectués à la troisième et cinquième semaine après le semis à raison de $150 \mathrm{~kg} / \mathrm{ha}$.

Le malathion $500 \mathrm{EC}$ à la dose de $1 \mathrm{~L} / \mathrm{Ha}$ a été utilisé comme insecticide pour la lutte contre les insectes. Toutes les pratiques culturales standard recommandées pour une bonne production ont été respectées.

\section{2-4. Collecte et analyse des données}

Plusieurs paramètres agronomiques ont été évalués :

La densité à la levée qui exprime le nombre de pieds levés est mesurée dans le carré d'observation $\left(1 \mathrm{~m}^{2}\right), 14$ jours après le semis.

La densité au tallage traduit le nombre de talles formées par une variété donnée. Elle est notée sur 5 touffes choisies au hasard également dans le carré d'observation, 35 jours après le semis.

Le nombre total de talles à maturité et le nombre de talles fertiles sont deux caractères 
agronomiques qui expriment le nombre total de talles produits à maturité et le nombre de talles portant des panicules. Les mesures sont faites simultanément sur 5 touffes choisies au hasard dans le carré d'observation. Le nombre de talles est compté et le nombre de talles fertiles est déterminé sur la même touffe, 70 jours après le semis.

La période de $50 \%$ de floraison est le nombre de jours après le semis qui correspond à la floraison de $50 \%$ des plants d'une variété sur une parcelle donnée. Cette date est rapportée à la date de semis de la parcelle pour déterminer le nombre de jours pour atteindre ce stade.

La taille des plantes à maturité qui indique la hauteur de la plante à la maturité est mesurée en cm sur 5 touffes choisies de façon aléatoire dans le carré d'observation. La taille est lue à l'extrémité de la panicule ou de la feuille la plus longue, 60 jours après le semis.

La verse caractérise les plants tombés au niveau de chaque variété au cours de l'expérimentation. La verse exprimée en pourcentage de plants tombés, est notée à la phase de maturité, 70 jours après le semis.

La pyriculariose est la maladie qui conduit à des pertes importantes de la production au niveau du riz pluvial en Afrique de l'Ouest. Cette maladie est notée à la phase de maturité, 70 jours après le semis avec l'échelle de notation suivante [6].

\begin{tabular}{cc} 
\% de plants infectés & Echelle de notation \\
\hline 0 & 0 \\
$1-10$ & 1 \\
$11-30$ & 3 \\
$31-50$ & 5 \\
$51-70$ & 7 \\
$71-100$ & 9
\end{tabular}

La production de grains d'une variété est obtenue à partir de la parcelle élémentaire. Pour une meilleure estimation de la production de grains ( $t / h a)$, le calcul est fait à partir de $14 \%$ du taux d'humidité.

Les analyses de variances ont été effectuées à l'aide de la procédure GLM (General Linear Model) du logiciel SAS [13].

Les moyennes des variétés ont été comparées par la méthode de la plus petite différence significative au seuil de $5 \%$.

\section{Résultats}

Le résultat d'analyse de variance (ANOVA) consigné dans le Tableau $I$ donne les valeurs des carrés moyens et leur niveau de signification pour les différents caractères agronomiques étudiés. Les variétés présentent une différence significative pour certains 
caractères et non significative pour d'autres indiquant qu'il existe une variabilité ou une homogénéité entre les variétés pour les caractères étudiés.

Tableau 1 : Analyse de la variance des caractères agronomiques mesurés sur les différentes variétés de riz testées.

\begin{tabular}{|c|c|c|c|c|c|c|c|c|}
\hline $\begin{array}{l}\text { Sources de } \\
\text { variation }\end{array}$ & $\begin{array}{l}\text { Degré de } \\
\text { liberté }\end{array}$ & $\begin{array}{l}\text { Densité à } \\
\text { la levée }\end{array}$ & $\begin{array}{l}\text { Densité } \\
\text { au tallage }\end{array}$ & $\begin{array}{l}\text { Nombre } \\
\text { total de } \\
\text { talles }\end{array}$ & $\begin{array}{l}\text { Nombre } \\
\text { de talles } \\
\text { fertiles }\end{array}$ & $\begin{array}{l}\text { Taille des } \\
\text { plantes à } \\
\text { maturité }\end{array}$ & $\begin{array}{l}\text { Période } \\
\text { de } 50 \% \\
\text { floraison }\end{array}$ & $\begin{array}{l}\text { Production } \\
\text { de grains }\end{array}$ \\
\hline $\begin{array}{l}\text { Répétitions } \\
\text { (blocs) }\end{array}$ & 2 & 1045.5 & 31743.4 & 103.4 & 23.4 & 3.4 & 30.3 & 32700.7 \\
\hline Erreur & 34 & 317.7 & 4900.4 & 2538.2 & 2229.5 & 56.0 & 4.4 & 19439.3 \\
\hline Variétés & 17 & $523^{\mathrm{ns}}$ & $7758.2^{\mathrm{ns}}$ & $7444.0 *$ & 6923.6 * & $1768.4^{* *}$ & $923.1^{* *}$ & $61807.3^{*}$ \\
\hline
\end{tabular}

\section{3-1. La densité à la levée}

L'analyse de variance montre qu'il existe une homogénéité entre les 18 variétés testées pour la densité à la levée. Le nombre de pieds levés, 14 jours après semis, varie entre 0 et 64 pieds au mètre carré (Tableau 2). Cependant, WAB 306-B-B- $L_{2}-L_{1}-H B$, WAB 99-12, WAB 99-2-I, WAB 450-I-B-P20-HB et WAB 365-B-6- $\mathrm{H}_{2}-\mathrm{HB}$ ont en moyenne une densité à la levée un peu plus importante par rapport aux autres variétés.

\section{3-2. La densité au tallage}

La comparaison des moyennes a permis de noter qu'il y a une homogénéité entre les variétés pour ce qui concerne la densité au tallage, déterminée à 35 jours après semis (Tableau 2). Mais, il est important d'indiquer que les variétés WAB 306-B-B- $\mathrm{L}_{2}-\mathrm{L}_{1}-\mathrm{HB}$, WAB 365-B-6- $\mathrm{H}_{2}-\mathrm{HB}$, WAB 99-2-1 et WAB 450-I-B-P20-HB ont une densité au tallage moyenne plus élevée que les autres variétés testées.

\section{3-3. Le nombre total de talles à maturité}

Le nombre total de talles évalué à 70 jours après semis varie, en moyenne, entre 0 et 276 talles au mètre carré (Tableau 2). Les variétés WAB 337-B-B-20-H4, WAB 365-B-6$H B$, WAB 450-24-1-B-P33-HB, WAB 99-H-16-HB et WAB 99-2-1 ont un nombre total de talles plus important. Ces variétés sont suivies par WAB 32-55 et WAB 306-B-B-L2-L1-HB avec 218 talles au mètre carré. 
Au niveau des NERICA, la variété NERICA 7 a donné, en moyenne, 14 et $16 \%$, respectivement, plus de talles au mètre carré que les variétés NERICA 2 et NERICA 4.

\section{3-4. Le nombre de talles fertiles}

L'analyse de variance indique qu'il existe une variabilité entre les 18 variétés testées au niveau du nombre total de talles fertiles déterminé à 70 jours après le semis.

Le nombre total de talles fertiles varie, en moyenne, entre 0 et 270 talles au mètre carré (Tableav 2). Les résultats obtenus montrent également que $90 \%$ des variétés ont entre 96 ef $100 \%$ de talles fertiles.

Tableau 2 : Evaluation du tallage des différentes variétés de riz testées de la levée à la maturité.

\begin{tabular}{|c|c|c|c|c|c|}
\hline Variétés & $\begin{array}{c}\text { Densité à la } \\
\text { levée } \\
\text { (pieds / } \mathrm{m}^{2} \text { ) }\end{array}$ & $\begin{array}{l}\text { Densité au } \\
\text { tallage } \\
\text { (pieds / } \mathrm{m}^{2} \text { ) }\end{array}$ & $\begin{array}{l}\text { Nombre total de } \\
\text { talles à maturité } \\
\text { (pieds } / \mathrm{m}^{2} \text { ) }\end{array}$ & $\begin{array}{l}\text { Nombre de } \\
\text { talles fertiles } \\
\text { (pieds } / \mathrm{m}^{2} \text { ) }\end{array}$ & $\begin{array}{c}\text { Pourcentage } \\
\text { de Talles } \\
\text { Fertiles (\%) }\end{array}$ \\
\hline$A B$ 99-12 & 61 & 203 & 202 & 202 & 100 \\
\hline WAB 450-24-1-P33-HB & 27 & 122 & 241 & 181 & 75 \\
\hline WAB 32-55 & 33 & 74 & 218 & 218 & 100 \\
\hline WAB 450-I-B-P24-HB & 26 & 99 & 168 & 168 & 100 \\
\hline WAB 224-12-HB & 40 & 108 & 156 & 153 & 98 \\
\hline WAB 450-I-B-P160-HB & 0 & 0 & 0 & 0 & 0 \\
\hline WAB 450-I-B-P91-HB & 30 & 105 & 176 & 175 & 99.7 \\
\hline WAB 450-I-B-P20-HB & 58 & 198 & 209 & 208 & 99.5 \\
\hline WAB 515-B-35A-1-1 & 48 & 111 & 201 & 201 & 100 \\
\hline WAB 306-B-B- $L_{2}-L_{1}-H B$ & 64 & 243 & 218 & 210 & 96 \\
\hline WAB 450-11-1-1-P31-1-HB & 35 & 88 & 181 & 177 & 97.8 \\
\hline WAB 450-11-1-1-P50-HB & 36 & 109 & 207 & 203 & 98 \\
\hline WAB 337-B-B-20-H4 & 36 & 130 & 276 & 269 & 97 \\
\hline WAB 570-10-1A-1-10 & 41 & 158 & 174 & 172 & 99 \\
\hline WAB 377-B-5-HI & 58 & 124 & 201 & 201 & 100 \\
\hline WAB 99-H-16-HB & 34 & 101 & 235 & 234 & 99.6 \\
\hline WAB 99-2-1 & 59 & 209 & 235 & 229 & 97 \\
\hline WAB 365-B-6- $\mathrm{H}_{2}-\mathrm{HB}$ & 54 & 233 & 269 & 265 & 97.8 \\
\hline Ppds (5 \%) & - & - & 106.3 & 99.6 & - \\
\hline CV $(\%)$ & - & - & 25.7 & 24.8 & - \\
\hline MG & 41.0 & 133.14 & 196 & 190 & 92 \\
\hline
\end{tabular}

Ppds (5\%) : Plus petite différence significative au seuil de $5 \%$

$\mathrm{CV}(\%) \quad$ : Coefficient de variation en pourcentage

$M G \quad$ : Moyenne générale 
Au niveau des variétés NERICA, NERICA 7 a également un nombre total de talles fertiles (208) plus élevé que NERICA 2 (177) et NERICA 4 (175). Cependant, ces 3 variétés ont produit un pourcentage presque équivalent de talles fertiles.

\section{3-5. La taille des plantes à maturité}

Les résultats obtenus ont permis d'indiquer qu'il existe une variabilité entre les variétés testées pour ce qui concerne la taille des plantes à maturité (Tableau 3). Ces résultats montrent également que $90 \%$ des variétés ont une taille à maturité supérieure à 100 $\mathrm{cm}$.

Les variétés WAB 450-I-B-P91-HB et WAB 377-B-5- $\mathrm{H}_{1}$ ont les tailles les plus importantes (135 et $136 \mathrm{~cm}$ ). WAB 32-55 et WAB 450-24-1-P33-HB ont une taille de 118 et $116 \mathrm{~cm}$ alors que WAB 337-B-B-20- $\mathrm{H}_{4}(86 \mathrm{~cm})$ a une taille inférieure à $100 \mathrm{~cm}$.

La variété WAB 450-24-I-B-P33-HB a produit, respectivement, $3.5 \%$ et $17 \%$ moins de grandes plantes par rapport aux variétés WAB 99-H-16-HB et WAB 450-I-B-P91-HB.

\section{3-6. La période de $50 \%$ de floraison}

II existe une variabilité entre les 18 variétés au niveau de la période de $50 \%$ de floraison. La période de $50 \%$ de floraison varie de 0 pour la variété à 100 jours après semis (Tableau 3).

Les variétés WAB 99-12, WAB 450-I-B-P20-HB, WAB 99-H-16-HB et WAB 450-11-1-1-P31-HB avec respectivement, $70,73,75$ et 77 jours ont été les plus précoces.

WAB 377-B-5- $\mathrm{H}_{1}$, WAB 32-55 et WAB 450-24-1-P33-HB sont intermédiaires avec une période de $50 \%$ de floraison, respectivement de 83,84 et 89 jours après semis. WAB 306-B-B-L2-LI-HB et WAB 224-12-HB représentent les variétés tardives avec une période de $50 \%$ floraison égale à 99 et 100 jours après semis.

\section{3-7. La verse}

Sur l'ensemble des variétés testées, seules six ont subi la verse (Tableau 3). Ce sont les variétés WAB 99-12, WAB 4506-I-B-P91-HB, WAB 450-I-B-P20-HB, WAB 450-11-1-1-P311-HB, WAB 99-H-16-HB et WAB 365-B6- $\mathrm{H}_{2}-\mathrm{HB}$ dont le taux de verse est compris entre 80 et $100 \%$. Les autres variétés comme WAB 99-12 et WAB 365-B-6- $\mathrm{H}_{2}-\mathrm{HB}$ qui ont un taux de verse autour de $10 \%$, ont été moins affectées.

\section{3-8. Le comportement des variétés face à des maladies}

Les observations sur le terrain ont montré que sur l'ensemble des variétés testées, sept ont été attaquées par la pyriculariose (Tableau 3). II s'agit des variétés WAB 450-I-B- 
P24-HB, WAB 450-I-B-P91-HB, WAB 450-I-B-P20-HB, WAB 450-11-1-1-P50-HB, WAB 570-10$1 A-1-10$ et WAB 99-2-1 dont le taux d'infection des plantes est compris entre 70 et $90 \%$.

\section{3-9. Le rendement}

Les résultats obtenus montrent qu'il existe une variabilité entre les 18 variétés pour le rendement. Les rendements observés sont très élevés et varient entre 0 et 7 tonnes à l'hectare (Tableav 3).

Tableau 3 : Evaluation des caractères agronomiques des différentes variétés de riz testées.

\begin{tabular}{lccccc}
\hline \multicolumn{1}{c}{ Variétés } & $\begin{array}{c}\text { Taille des } \\
\text { plantes à } \\
\text { maturité (cm) }\end{array}$ & $\begin{array}{c}\text { Période } \\
50 \% \\
\text { floraison (i) }\end{array}$ & $\begin{array}{c}\text { Notes de la } \\
\text { verse (\%) }\end{array}$ & $\begin{array}{c}\text { Notes de } \\
\text { pyriculariose }\end{array}$ & $\begin{array}{c}\text { Production } \\
\text { de grains } \\
(\dagger / \text { ha })\end{array}$ \\
\hline WAB 99-12 & 102 & 70 & 100 & 1 & 2.87 \\
WAB 450-24-1-P33-HB & 116 & 89 & 0 & 3 & 7.03 \\
WAB 32-55 & 118 & 84 & 0 & 3 & 5.10 \\
WAB 450-I-B-P24-HB & 117 & 89 & 0 & 7 & 3.40 \\
WAB 224-12-HB & 121 & 100 & 0 & 1 & 2.30 \\
WAB 450-I-B-P160-HB & 0 & 0 & 0 & 1 & 0 \\
WAB 450-I-B-P91-HB & 136 & 85 & 100 & 9 & 5.40 \\
WAB 450-I-B-P20-HB & 127 & 73 & 100 & 9 & 4.06 \\
WAB 515-B-35A-1-1 & 121 & 87 & 0 & 1 & 3.00 \\
WAB 306-B-B-L2-L -HB & 118 & 99 & 0 & 9 & 1.42 \\
WAB 450-11-1-1-P31-1-HB & 108 & 77 & 100 & 3 & 5.10 \\
WAB 450-11-I-1-P50-HB & 112 & 83 & 0 & 7 & 2.63 \\
WAB 337-B-B-20-H4 & 121 & 87 & 0 & 3 & 3.00 \\
WAB 570-10-1A-1-10 & 110 & 89 & 0 & 9 & 2.70 \\
WAB 377-B-5-HI & 135 & 83 & 0 & 1 & 4.30 \\
WAB 99-H-16-HB & 120 & 75 & 100 & 1 & 6.64 \\
WAB 99-2-1 & 119 & 88 & 0 & 7 & 3.04 \\
WAB 365-B-6-H - HB & 86 & 72 & 100 & 1 & 4.20 \\
\hline Ppds (5 \%) & 15.8 & 4.4 & - & - & 294.2 \\
CV (\%) & 6.8 & 2.7 & - & - & 38.1 \\
MG & 110 & 79 & - & - & 3.66 \\
\hline
\end{tabular}

Ppds (5\%) : Plus petite différence significative au seuil de $5 \%$

CV(\%) : Coefficient de variation en pourcentage

MG : Moyenne générale

WAB 450-24-1-B-P33-HB (7t/ha), WAB 99-H-16-HB (6t/ha), NERICA 4 (5.40t/ha) et NERICA 2 et WAB 32-55 (5t/ha) ont été les variétés les plus performantes. Les variétés WAB 306-B- 
B-20- $\mathrm{H}_{4}(1.40 \mathrm{t} / \mathrm{ha})$, WAB 224-12-HB (2.30t/ha), WAB 450-11-1-1-P50-HB (2.63t/ha), WAB 570-10-1A-1-10 (2.70t/ha) et WAB 99-12 (2.87t/ha) ont été les moins productives.

La variété WAB 450-24-I-B-P33-HB a produit, respectivement, $5.6 \%$ et $56.8 \%$, plus de grains que WAB 99-H-16-HB et WAB 99-12.

\section{Discussion}

Notre étude a permis d'identifier un groupe de variétés ayant produit un nombre important de talles au mètre carré. Dans ce groupe, les variétés WAB 337-B-B-20- $\mathrm{H}_{4}$, WAB 365-B-6- ${ }_{2}-H B$, WAB 450-24-I-B-P33-HB et WAB 99-12 ont présenté en plus un bon développement végétatif, ce qui permet d'indiquer que ces variétés pourraient produire un nombre élevé de talles fertiles. Il est à noter également que le nombre total de talles produits par une variété est lié au stade de développement des plantes et strictement lié à la variété.

Les résultats obtenus ont montré que $90 \%$ de toutes les variétés testées ont un nombre important de talles fertiles en rapport avec le nombre total de talles produits. Ainsi, les variétés WAB 32-55, WAB 377-B-5- H $_{1}$ et NERICA 7 ont donné entre 75 et $100 \%$ de talles fertiles. Les résultats obtenus avec ces variétés améliorées sont similaires à ceux observés antérieurement en Afrique de l'Ouest indiquant que ces nouvelles variétés peuvent produire autour de $100 \%$ de talles fertiles [14].

II est important de noter que le rendement n'est pas directement corrélé au développement végétatif de la plante [15]. Cependant, il est probable que les variétés qui produisent plus de talles fertiles pourraient avoir un rendement élevé.

Les résultats montrent que $90 \%$ des variétés testées ont présenté une taille moyenne supérieure à $100 \mathrm{~cm}$. Ces résultats observés avoisinent la taille moyenne de $120 \mathrm{~cm}$ obtenue avec ces mêmes variétés améliorées dans des essais conduits dans différentes conditions environnementales [16].

La grande taille observée dans l'ensemble peut être due aux facteurs environnementaux comme le climat et à la bonne activité biologique du sol qui ont entraîné un bon développement végétatif des plantes. II est à noter également que la grande taille de ces nouvelles variétés faciliterait la récolte manuelle au niveau des paysans [17].

La notation de la date de floraison renseigne sur la durée du cycle végétatif des variétés parce que la période entre la floraison et la maturité est généralement constante. Les résultats obtenus indiquent que la plupart des variétés testées ont été précoces avec une période de $50 \%$ de floraison autour de 70 jours en moyenne. Le même phénomène a été observé en Afrique de l'Ouest avec ces nouvelles variétés améliorées qui arrivent à maturité entre 90 et 100 jours et présentent ainsi une précocité à la maturité de 30 à 50 
jours par rapport aux variétés typiques de riz pluvial et les variétés améliorées demi naines d'Afrique [18]. En effet, les variétés typiques de riz pluvial arrivent à maturité entre 150 et 170 jours et les variétés améliorées demi naines entre 120 et 140 jours après semis [16]. La différence entre ces variétés peut être attribuée à l'amélioration génétique des nouvelles variétés de riz pluvial qui présentent des performances de plus en plus importantes.

Les résultats obtenus montrent que certaines variétés comme par exemple NERICA 2, NERICA 4, NERICA 7, WAB 99-H-16-HB ont été affectées par la verse alors que ces mêmes variétés n'avaient pas été affectées dans les essais menés antérieurement en Afrique de l'Ouest [16].

Comme ces variétés ont présenté dans les deux situations, des développements végétatifs similaires, la verse observée au cours de cette expérimentation peut être attribuée à la dose d'engrais et aux grands vents survenus à la fin de la phase de maturation qui ont fragilisé surtout les plantes à grande taille.

Plusieurs variétés ont été affectées par les maladies comme la pyriculariose et confirme ainsi les observations précédentes qui ont indiqué que la pyriculariose est une maladie qui apparaît dans presque toutes les zones de production de riz [3].

La pyriculariose est l'une des maladies les plus importantes du riz pluvial en Afrique de l'Ouest parce qu'elle peut conduire à des pertes importantes de production [17].

Cependant, il est à noter que ces maladies observées seulement à la fin de la phase de maturation surtout au moment de la saison sèche, n'ont eu aucune incidence sur les caractères agronomiques des différentes variétés testées. Ceci montre que l'importance de la maladie peut être aussi fonction de la pluviométrie de la région.

Les variétés testées dans notre étude sont les mêmes qui ont exprimé des productions moyennes en grains de 4 tonnes à l'hectare dans les essais menés précédemment en Afrique de l'ovest [3]. Ces variétés ont permis une augmentation de la production de $50 \%$ par rapport aux variétés traditionnelles de riz pluvial qui produisent 2 tonnes à l'hectare. Pour ces auteurs, les productions en grains obtenues étaient dues en partie au grand nombre de panicules que présentaient ces variétés. Ils ont indiqué que ces rendements pourraient être supérieurs dans les conditions optimales de production [19].

Les résultats observés dans notre étude ont permis d'identifier un groupe de variétés avec un rendement assez élevé atteignant même 7 tonnes à l'hectare. Le rendement élevé de ces variétés, observé dans cette région peut être dû aux bonnes conditions de production tel que le climat et aussi à la bonne activité biologique du sol qui ont conduit à un bon développement des plantes. 


\section{Conclusion}

L'objectif principal de cette étude a été de sélectionner des variétés de riz pluvial les plus performantes parmi un lot de 18, dans la région Nord de la République du Congo Brazzaville. Les résultats observés à partir de l'évaluation agronomique ont montré qu'il existe une variabilité entre ces différentes variétés testées.

Cette variabilité a été observée pour les caractères comme le nombre total de talles à maturité, le nombre total de talles fertiles, la taille des plantes à maturité, la période de $50 \%$ de floraison, la verse, le comportement des plantes face à la pyriculariose et le rendement.

Les analyses de variance réalisées au niveau de ces paramètres agronomiques ont mis en évidence que $90 \%$ des variétés testées ont entre 96 et $100 \%$ de talles fertiles et une taille supérieure à $100 \mathrm{~cm}$. Ces résultats ont également montré que $70 \%$ environ des variétés ont un rendement supérieur ou égal à $3 \mathrm{t} / \mathrm{ha}$.

Sur la base des caractères agronomiques évalués, seulement trois ont pu être sélectionnées parmi le lot de 18. Ce sont les variétés WAB 450-24-I-B-P33-HB, WAB 377$B-5-H_{1}$ et WAB 32-55 qui ont en plus un rendement respectif de $7.03,4.30$ et $6.10 \mathrm{t} / \mathrm{ha}$, un taux d'infection autour de $10 \%$ et n'ont pas subi de verse.

\section{Remerciements}

Les auteurs souhaitent remercier ENI Congo pour sa contribution à la réalisation de ce projet riz dans le district de OYO, en république du Congo Brazzaville.

\section{Références}

[1] - A. Gallais et H. Bannerot. "Amélioration des espèces cultivées". INRA Paris Editions (1992) 71-88.

[2] - W. Zhang, Y. Qi and Y. Liv, "Forecasting Trend of Rice Production of the World and Regions. New directions for a diverse planet ": Proceedings of the $4^{\text {th }}$ International Crop Science Congress, Brisbane, Australia, 26 sep-1 October (2004). www.cropscience.org.av

[3] - G. R. Guéi, F. Abamu, K. Traoré and S. Naman, "Genetic variability in morphological and physiological traits within and among rice species and their interspecific progenies". Agronomie Africaine, 16 (1) (2003) 15-32.

[4] - IRRI (1996). Standard Evaluation System for Rice. $4^{\text {th }}$ edition. International Rice Research Institue Los Banos, Philippines.

[5] - FAO (2004). Food and Agriculture Organization of the United Nations. International Year of Rice 2004. Rice is life. http://www.fao.org./rice 2004.

\section{A. S. P. Nguetta et al.}


[6] - M. Jacquot, G. Clément, A. Ghesquière, J. C. Glaszmann, E. Guiderdoni, et D. Tharreau. Les riz. in : L'amélioration des plantes tropicales. CIRAD et ORSTOM, (1997) $533 \mathrm{p}$.

[7] - A. Angladette, "Le Riz : Techniques agricoles et productions tropicales". Editions GP. Maisonneuve et Larose, (1966) $17 \mathrm{p}$.

[8] - ADRA0, "Rice trends in sub-Saharan Africa. A synthesis of statistics on rice production, trade and consumption", (1996) 1973-92. West Africa Rice Development Association, BP 2551, Bouaké 01, Côte d'Ivoire.

[9] - DSAP. "Direction de la Statistique Appliquée. Grands axes de la politique agricole $d u$ Congo. Mesure de relance de la production agricole", (1999).

[10] - Kormawa P., K. Shellemiah, and A. Touré, "Developments and future prospects for rice research and production in Africa", Agronomie Africaine, numéro spécial, (5) (2005) $1-16$.

[11] - Rapport National du Congo. Sommet National de l'Alimentation. Direction de la Statistique Appliquée (1996).

[12] - ADRAO, Association pour le Développement de la Riziculture en Afrique de l'Ouest, M'bé, Bouaké, Côte d'Ivoire. Rapport de mission de prospection au Congo Brazzaville, (2003) pp 1-5.

[13] - SAS, "Guide for personal computers, version 8 edition", Cary, NC, SAS institute Inc, (1999) $1028 \mathrm{p}$.

[14] - P. J. Monty, S. Mandé and G. K. Aluko, "Diversity and potential of Oryza glaberrima steud. In upland rice breeding", Breeding Science, (47) (1997) 395-398.

[15] - G. R. Guéi, K. A. Sanni, F. S. Abamu, and I. Fawolé, "Genetic diversity of rice(Oryza sativa L.) landraces from Côte d'Ivoire", Agronomie Africaine, numéro special, (5) (2005) 17-28.

[16] - P. J. Monty, M. Dingkhun, G. K. Aluko and S. Mandé, "Interspecific Oryza sativa L. x O. glaberrima steud. progenies in upland rice improvement". Euphytica, (92) (1997) 237-246.

[17] - ADRA0, Association pour le Développement de la Riziculture en Afrique de l'Ouest. "Bintu and Her New Rice for Africa. Breaking the shackles of the slash - burn farming In the world's poorest region", (2001) p. 6-13.

[18] - A. S. P. Nguetta, R. G. Guéi et S. Diatta, "Contribution à l'identification de variétés performantes de riz inondé (Oryza sp.) dans la région subéquatoriale du Congo Brazzaville". Afrique Science, 01 (1) (2005) 81-93. www.afriquescience.org

[19] - K. Futakuchi, S. Tobita, S. Diatta and A. Audebert, "WARDA's work on theNew Rice for Africa (NERICA), interspecific Oryza sativa L. x 0. glaberrima steud. Progenies", Japanese Journal of Crop Science, (Extra issue), 72 (1) (2003) 324-325. 